Int. J. of Bif. and Chaos

\title{
Transition to Chaotic Phase Synchronization through Random Phase Jumps
}

\author{
D. Pazó*, I.P. Mariño, V. Pérez-Villar and V. Pérez-Muñuzuri \\ Group of Nonlinear Physics, Faculty of Physics, \\ University of Santiago de Compostela, \\ 15706 Santiago de Compostela, Spain
}

(November 15, 2018)

\begin{abstract}
Phase synchronization is shown to occur between opposite cells of a ring consisting of chaotic Lorenz oscillators coupled unidirectionally through driving. As the coupling strength is diminished, full phase synchronization cannot be achieved due to random generation of phase jumps. The brownian dynamics underlying this process is studied in terms of a stochastic diffusion model of a particle in a one-dimensional medium.
\end{abstract}

*E-mail: diego@fmmeteo.usc.es; http://fmmeteo.usc.es 


\section{INTRODUCTION}

Phase synchronization phenomena in coupled chaotic systems have been extensively studied during the last few years in the context of non-identical chaotic systems (Rosenblum et al., 1996; Osipov et al., 1997), ecological systems (Earn et al., 1998; Blasius et al., 1999), physiological systems (Schäfer et al., 1998), chaotic systems forced by an external periodic or noisy signal (Pikovsky et al., 1997a, 1997b), an ensemble of coupled chaotic oscillators (Pikovsky et al., 1997c; Osipov et al., 1997), and with an electronic model of two Rössler oscillators (Parlitz et al., 1996). This effect owes its name from the classical definition of synchronization of periodic oscillators which is described in terms of locking or entrainment of the phases, while the amplitudes can be quite different. Hence, synchronization of chaotic oscillators can be defined in the most general case, as the locking between the phases of two coupled systems, while the amplitudes remain chaotically varying in time (Rosenblum et al., 1996).

For chaotic oscillators, there is no unique definition of phase. An approach to determine the amplitude $A$ and phase $\phi$ of a narrow-band signal $s(t)$ is based on the analytic signal concept that considers an analytical signal $\psi(t)$ as a complex function of time, $\psi(t)=$ $s(t)+\imath \tilde{s}(t)=A(t) e^{\imath \phi(t)}$ and $\tilde{s}(t)$ is the Hilbert transform of $s(t)$ (Rosenblum et al., 1996). However, in other cases phase and amplitude can be defined as a function of the natural variables of the oscillator. For example, for the Rössler attractor $\phi=\arctan (y / x)$ (Pikovsky et al., 1997d) or $\phi=\arctan \left(y / \sqrt{x^{2}+y^{2}}\right)$ (Rosa et al., 1998), and for the Lorenz model $\phi=$ $\arctan \left[\left(\sqrt{x^{2}+y^{2}}-u_{0}\right) /\left(z-z_{0}\right)\right]$ (Pikovsky et al., 1997b), where $u_{0}$ and $z_{0}$ are constants.

In this paper, we focus our interest in the phenomenon of phase synchronization between chaotic Lorenz systems coupled unidirectionally through driving in a ring geometry. It has been shown, that for an appropriate set of parameters, a ring of $N$ coupled Lorenz systems shows a Periodic Rotating Wave (PRW) where neighboring oscillators exhibit a phase difference of $2 \pi / N$ and the amplitude varies with time sinusoidally (Mariño et al., 1998). This system, with a different set of parameters also exhibits Chaotic Rotating Waves (CRW) defined as well by a phase difference between neighboring cells of $2 \pi / N$ but the amplitude remains chaotic (Sánchez and Matías, 1999). In this structure there exists a superposition of Fourier modes $k=0$ and $k=1$. Here we will show a transition from a 
PRW with a phase difference of $2 \pi / N$ to a CRW with a phase difference of $4 \pi / N$, where opposite cells are phase synchronized ( $N$ even). Depending on the unidirectional coupling strength, random/brownian $2 \pi$-phase slips develop during the mentioned transition.

\section{MODEL}

We shall consider rings of Lorenz attractors coupled in such a way that the dynamical behavior (Güémez and Matías, 1995) is defined by,

$$
\begin{aligned}
\dot{x}_{j} & =\sigma\left(y_{j}-x_{j}\right) \\
\dot{y}_{j} & =R\left(\beta x_{j-1}+(1-\beta) x_{j}\right)-y_{j}-x_{j} z_{j} \\
\dot{z}_{j} & =x_{j} y_{j}-b z_{j}
\end{aligned}
$$

with $\sigma, R$ and $b$ positive parameters. Usual parameters values are $\sigma=10, b=\frac{8}{3}$ and $R=28$. In Eq. (1), $\beta$ accounts for the coupling strength, $j$ runs from 1 to $N$ (number of cells in the array), and for $j=1, x_{0}=x_{N}$.

For $\beta=1$, it was observed (Matías et al., 1997; Mariño et al., 1998) that the synchronized chaotic state is stable if the size of the ring is small enough $N=2$, while for a certain critical number $N_{c}=3$ in the case of the Lorenz model, an instability associated to the first Fourier mode $k=1$ destroys the uniform chaotic state, leading to a PRW. As the size of the ring is increased, new Fourier modes become unstable and for $N=6$ a second instability $(k=2)$ develops that could lead to a Chaotic Rotating Wave (CRW) where neighboring oscillators exhibit a phase difference of $4 \pi / N$ as it is shown in Fig. 1)(a), that is, Fourier modes $k=1$ and $k=2$ compete in a nonlinear way. Thus, opposite cells are phase synchronized while amplitudes remain chaotic and are, in general, uncorrelated. Figure [(b) shows the uncorrelated values of the amplitudes of $x_{j+N / 2}(t)$ as a function of $x_{j}(t)$. Since the second Fourier mode plays an important role for phase synchronization, we will focus our study in a ring consisiting of $N=6$ Lorenz cells described by Eq. (1) as $\beta$ is varied. This phase synchronization describes the onset of long-range correlations in chaotic oscillations (suppression of phase diffusion), and thus also corresponds to the appearance of certain order inside chaos that here is shown as a CRW with certain similarities to a quasiperiodic motion. 
To study phase synchronization of coupled chaotic systems, we calculate the phases of the oscillators and then check whether the weak locking condition $\Delta \phi=\left|n \phi_{j}-m \phi_{j+N / 2}\right|<$ const is satisfied. In this paper, we restrict ourselves to the case of $m=n=1$. The definition of the phase for a given oscillator may be a problematic task as soon as there is not a center of rotation. Fig. 2 shows the $(x, y)$ projection of an oscillator phase space for two $\beta$ values, $\beta=1.0$ and $\beta=0.85$. At $\beta=1.0$ a center of rotation can be clearly distinguished at $(x, y)=(0,0)$. Then a Poincaré surface of section $y=x, x>0$ allows us to define the phase as (Pikovsky et al., 1997b)

$$
\phi(t)=n+\frac{t-t_{n}}{t_{n+1}-t_{n}}
$$

where $t_{n}$ is the nth crossing of the surface. Note that the phase has been normalized by a factor $2 \pi$. We see that with the surface chosen in Fig. 2(a) crossings are equal to maxima of the variable $x(t)$. Therefore, we can know at what times the phase is an integer just looking at the time evolution of the variable $x$, this criterion has been used before (Blasius et al., 1999). As long as $\beta$ decreases unproper rotations become more frequent, (see Fig. 2(b)). However it is clear the existence of a "rotation axis", unlike the funnel Rössler attractor case (see e.g. Pikovsky et al., 1997b) where an independent center of rotations emerges at one side of the attractor. In consequence phase is increased in one unit (i.e. $2 \pi$ radians) when an unproper rotation occurs. As it will be later discussed the time difference of two consecutive maxima does not depend on the own nature of each rotation. So it seems that our definition provides a "good" period. The instantaneous phase $\phi(t)$ will be determined through linear interpolation after calculating the instants of time at which maxima appear in the $x(t)$ series.

\section{RESULTS}

The main effect of varying $\beta$ is shown in Fig. 3. As shown above, for $\beta=1$ opposite oscillators within the ring are phase synchronized. As $\beta$ decreases, opposite cells still remain phase synchronized, except for some phase jumps. These events are defined as the nonoccurrence of a maxima at its due time in one of the $x(t)$ signals corresponding to cells $j$ or $j+N / 2$. In other words, we will assume that a phase jump occurs if $\Delta \phi(t)=\phi_{j}(t)-\phi_{j+N / 2}(t)$ 
changes by approximately \pm 1 for two consecutive maxima of $x(t)$. Note for example for $\beta=0.76$ and $\beta=0.85$ two jumps are encircled in Fig. 3. Unproper rotations are signaled, it is clear that they do not produce phase slips, although both phenomena appear when the signal turns more chaotic. Further decreasing the coupling strength $\beta$ finally leads to the formation of a PRW with a phase difference of $1 / 6(\bmod 1)$ between neighboring cells. The transition between PRWs and phase synchronization with jumps occurs for a critical value of $\beta_{c} \approx 0.75$.

The distribution of phase jumps is shown in the sequence of figures at the rightside of Fig. 3 where the periods of time $T$ between consecutive maxima of $x(t)$ are shown for two opposite cells within the ring. As $\beta$ is decreased, the map $T_{j+N / 2}$ as a function of $T_{j}$ shows a greater dispersion from the mean value (located in the center of the figures) until the critical value $\beta_{c}$ is reached. For high values of $\beta$, a small deviation of periods around the mean value appears according to the way the phase synchronization has been defined $\left(\left|\phi_{1}-\phi_{4}\right|<\right.$ const $<1 / 2$ ). As the value of $\beta$ decreases, the dispersion around the mean value increases at the same time that two independent accumulation regions responsible for the phase slips appear (see circles at the rightside of Fig. 3). Notice that these phase slips are not related to the unproper rotations which are represented by maxima (minima) peaks of the temporal serie that do not take a positive (negative) value (see arrows in the leftside of Fig. 3). The process of phase jumps formation is as follows; consecutive $x(t)$ maxima of one cell remains phase synchronized with the opposite cell within the ring, until a phase jump occurs spontaneously, which corresponds to jumps from arms numbered (2) and (3) in Fig. 3 to the encircled zones. That is, phase slips are characterized by the sequences $2 \rightarrow 1 \rightarrow 2$ and $3 \rightarrow 4 \rightarrow 3$. At the same time, fluctuations in $\Delta \phi$ (i.e. no perfect synchronization between maxima) leads to jumps between zones (2) and (3). Besides, it must be pointed out that as $\beta$ is decreased the concept of phase synchronization defined above and used here becomes less restrictive as the dispersion around the mean value increases (const $\rightarrow 1 / 2$ ).

The number of phase slips occurring at a given interval of time decreases as $\beta$ is increased. Then, when opposite cells within the ring are phase synchronized the phase difference $|\Delta \phi(t)|$ is, on average, constant in time. But, if phase slips occur for $\beta_{c}<\beta<1$, then one would expect that for $M$ different initial conditions, the averaged square phase difference dynamics 
will be generally diffusive, so for large $t$,

$$
\left\langle|\Delta \phi(t)|^{2}\right\rangle=2 D t
$$

where $D$ is the diffusion constant. Figure 1 shows the linear dependence found for the root mean square of the phase difference $\left\langle|\Delta \phi(t)|^{2}\right\rangle^{1 / 2}$ as a function of time, in a log-log plot for $M=14$ different random initial conditions for the Lorenz cells within the ring and for four different values of $\beta$. The four graphs fit to a straight line with slope $S \approx 1 / 2$ as expected from Eq. (3) (see Table I for the fitted values).

The distribution of temporal periods $\tau$ between two consecutive phase jumps is shown in Fig. 5 for two different values of $\beta$. Note the occurrence of longer periods of time $\tau$ for higher values of $\beta$. These distributions show an exponential decay with $\tau$ as a consequence of the intrinsic random/brownian nature of the dynamical process underlying the formation of phase slips. Moreover, neither the phase jumps occur simultaneously for all couples of opposite cells within the array, nor the phase jumps are correlated in space, which is in agreement with the random dynamics of phase slip formation.

\section{DISCUSSION}

From Fig. 5 a mean value of the period $\langle\tau\rangle$ for each value of $\beta$ can be defined. Now, by using a simple model of stochastic diffusion of a particle in a one-dimensional medium (random discrete walk), the averaged quadratic dispersion from the phase synchronized state $(\Delta \phi \approx 0)$ is given by the following equation,

$$
\left\langle|\Delta \phi(t)|^{2}\right\rangle=\frac{t}{\langle\tau\rangle}
$$

where $t /\langle\tau\rangle$ is the number of phase jumps that have appeared for $t \gg\langle\tau\rangle$. Consequently, comparing Eqs. (3) and (4), it is possible to calculate a theoretical value for the diffusion coefficient $D_{t h}=(2\langle\tau\rangle)^{-1}$. A comparison between the diffusion coefficient $D_{\exp }$ obtained after fitting the log-log plots given in Fig. 囵and $D_{t h}$ is shown in Table I. Note the good agreement between both coefficients for large values of $\beta$ as expected for a typical brownian dynamics. It must be noted that $\langle\tau\rangle$ increases dramatically with $\beta$ (see Fig. 5 and the values of $D_{t h}$ in Table I) in such a way that it is not possible to assure the existence of an upper 
limit of $\beta$ above it no jumps appear. For $\beta \rightarrow \beta_{c}$ we have found small values of $\langle\tau\rangle$ of the order of the mean period between two consecutive maxima of $x(t)$. Thus, jumps occur frequently in time and a random, uncorrelated in time, sequence cannot be assured (the system shows a tendence to display $+1,-1,+1, \ldots$ slips series). Then, the obtained values of the diffusion $D_{\text {exp }}$ are smaller than those predicted $D_{t h}$ using $\langle\tau\rangle$.

The transition between periodic rotating waves and phase synchronized chaotic rotating waves has been shown to occur as the coupling strength $\beta$ is increased. For values of $\beta>\beta_{c}$, phase slips develop randomly in time following a diffusive process given by Eq. (3). Note that the dynamics of the phase defined for a single chaotic oscillator is generally diffusive as well (Pikovsky et al., 1997b) and in this case, $D$ determines the phase coherence of the chaotic oscillations which is inversely proportional to the width of the spectral peak of the chaotic attractor. On the other hand, for coupled unsynchronized nonidentical chaotic oscillators the average phase difference grows linearly with time (Blasius et al., 1999). Nevertheless, we have shown a different behavior where the root mean square of the phase difference grows with $t^{1 / 2}$ as a consequence of phase slips random formation.

\section{ACKNOWLEDGEMENTS}

We want to thank I. Sendiña-Nadal for fruitful discussions and comments on this work. The support by DGES and Xunta de Galicia under Research Grants PB97-0540 and XUGA20602B97, respectively, is gratefully acknowledged.

\section{REFERENCES}

Blasius, B., Huppert, A. and Stone L. [1999] "Complex dynamics and phase synchronization in spatially extended ecological systems". Nature 399, 354-359.

Earn, D.J. D., Rohani, P. and Grenfell, B. [1998] "Persistence, chaos, and synchrony in ecology and epidemiology". Proc. R. Soc. Lond. B 265, 7-10.

Güémez, J. and Matías, M.A. [1995] "Modified method for synchronizing and cascading chaotic systems". Phys. Rev. E 52, 2145-2148. 
Mariño, I.P., Pérez-Muñuzuri, V. and Matías, M.A. [1998] "Desynchronization transitions in rings of coupled chaotic oscillators". Int. J. of Bif. and Chaos 8, 1733-1738.

Matías, M.A., Pérez-Muñuzuri, V. Lorenzo, M.N., Mariño, I.P. and Pérez-Villar, V. [1997] "Observation of a fast rotating wave in rings of coupled chaotic oscillators". Phys. Rev. Lett. 78, 219-222.

Osipov, G.V., Pikovsky, A.S., Rosenblum, M.G. and Kurths J. [1997] "Phase synchronization effects in a lattice of nonidentical Rössler oscillators". Phys. Rev E 55, 2353-2361.

Parlitz, U., Junge, L., Lauterborn, W. and Kocarev, L. [1996] "Experimental observation of phase synchronization". Phys. Rev. E 54, 2115-2117.

Pikovsky, A., Osipov, G., Rosenblum, M., Zaks, M. and Kurths, J. [1997a] "Attractorrepeller collision and eyelet intermittency at the transition to phase synchronization". Phys. Rev. Lett. 79, 47-50.

Pikovsky, A., Rosenblum, M., Osipov, G., and Kurths, J. [1997b] "Phase synchronization of chaotic oscillators by external driving". Physica D 104, 219-238.

Pikovsky, A., Rosenblum, M.G. and Kurths, J. [1997c] "Synchronization in a population of globally coupled chaotic oscillators". Europhys. Lett. 34, 165-170.

Pikovsky, A., Zaks, M., Rosenblum, M., Osipov, G. and Kurths, J. [1997d] "Phase synchronization of chaotic oscillations in terms of periodic orbits". Chaos 7, 680-687.

Rosa, E., Ott, E. and Hess, M.H. [1998] "Transition to phase synchronization of chaos". Phys. Rev. Lett. 80, 1642-1645.

Rosenblum, M.G., Pikovsky, A.S. and Kurths, J. [1996] "Phase synchronization of chaotic oscillators". Phys. Rev. Lett. 76, 1804-1807.

Sánchez, E., Matías, M.A. [1999] "Transition to rotating chaotic waves in arrays of coupled Lorenz oscillators". Int. J. of Bif. and Chaos 9 (in press).

Schäfer, C., Rosenblum, G.R., Kurths, J. and Abel, H.H. [1998] "Heartbeat synchronized with ventilation". Nature 392, 239-240. 


\section{FIGURES}

FIG. 1. Temporal evolution of the variable $x$ of three contiguous Lorenz cells coupled unidirectionally through driving within a ring of $N=6$ oscillators and $\beta=1$ (a). The figure shows a Chaotic Rotating Wave (CRW) with approximate phase relationship of $4 \pi / N$. Variables $x_{j}$ and $x_{j+N / 2}(j=1)$ are then phase correlated although their amplitudes remain chaotic, and uncorrelated (b).

FIG. 2. Projections onto the $x-y$ plane of the trajectory followed by a chaotic oscillator when considering an array of six identical cells coupled with different values of the coupling parameter: (a) $\beta=1.0$ and (b) $\beta=0.85$. In (a) the straight line indicates the Poincaré surface of section $y=x, x>0$, while in (b) it represents the "rotation axis", since now a rotation center is not properly defined.

FIG. 3. Temporal evolution of the variables $x_{1}$ and $x_{4}$, corresponding to two opposite cells within a ring of $N=6$ Lorenz oscillators, (leftside) and temporal period $T_{4}$ between maxima of $x_{4}(t)$ as a function of $T_{1}$ (rightside) for four different values of $\beta$. Note in the leftside of the figure that the phase slips are encircled. For $\beta=0.90$ the arrows indicate the time intervals where the phase is not properly defined (here the local minima are positive). Note that these positive minima are not related to the encircled phase slips. At the rightside, circles mark those accumulation regions where the periods are greater than the average value, signalling then the occurrence of a phase jump. See text for an explanation of the sequence of numbers: $1 \rightarrow 4$. For $\beta=0.75$ the arrow indicates the position of a single dot corresponding to the value of $T_{1}=T_{4}=0.36$ t.u.

FIG. 4. Log-log plot of the root mean square of the phase difference $\left\langle|\Delta \phi|^{2}\right\rangle^{1 / 2}$ as a function of time for four different values of the coupling strength $\beta$. Lines correspond to a linear fitting.

FIG. 5. Distribution of temporal periods $\tau$ between two phase jumps for two different values of $\beta$. Note the different time scales between both graphs. Fitting lines correspond to an exponential decay (first order in time). 


\begin{tabular}{|c|c|c|c|}
\hline \hline$\beta$ & $S$ & $D_{\text {exp }} \times 10^{-3}\left(\mathrm{t} . \mathrm{u} .^{-1}\right)$ & $D_{t h} \times 10^{-3}\left(\mathrm{t} . \mathrm{u} .^{-1}\right)$ \\
\hline 0.85 & $0.51 \pm 0.02$ & $3.53 \pm 2.35$ & $4.41 \pm 0.05$ \\
0.86 & $0.47 \pm 0.01$ & $2.98 \pm 1.09$ & $2.32 \pm 0.03$ \\
0.87 & $0.48 \pm 0.02$ & $1.55 \pm 1.02$ & $0.73 \pm 0.02$ \\
0.88 & $0.52 \pm 0.01$ & $0.050 \pm 0.018$ & $0.055 \pm 0.001$ \\
0.89 & $0.48 \pm 0.01$ & $0.006 \pm 0.002$ & $0.004 \pm 0.001$ \\
\hline \hline
\end{tabular}

TABLE I: Values of the slope $S$, and $D_{\text {exp }}$ obtained from the linear fitting of Fig. 4 and Eq. (3). $D_{t h}=(2\langle\tau\rangle)^{-1}$ is calculated from the mean values of $\tau$. 
This figure "fig1.jpg" is available in "jpg" format from: http://arxiv.org/ps/chao-dyn/9912026v1 
This figure "fig2.jpg" is available in "jpg" format from: http://arxiv.org/ps/chao-dyn/9912026v1 
This figure "fig3.jpg" is available in "jpg" format from: http://arxiv.org/ps/chao-dyn/9912026v1 
This figure "fig4.jpg" is available in "jpg" format from: http://arxiv.org/ps/chao-dyn/9912026v1 
This figure "fig5.jpg" is available in "jpg" format from: http://arxiv.org/ps/chao-dyn/9912026v1 